\title{
DEVELOPMENT OF A RF BIOSENSOR DESIGN TO DETECT CHANGES IN SCATTERING PARAMETERS OF AQUEOUS MATERIALS DURING RADIO FREQUENCY WAVE EXPOSURE
}

\author{
Mary Pearson, Daniel Ewert, Ryan Striker, and Benjamin Braaten \\ North Dakota State University, Department of Electrical and Computer Engineering \\ 1411 Centennial Blvd, Fargo, ND 58105
}

Corresponding Author: Mary Pearson

Email: mary.pearson@ndsu.edu

https://doi.org/10.34107/BiomedSciInstrum.57.04290

\begin{abstract}
The advancing field of biosensor design continues pushing for smaller, inexpensive, yet accurate sensor designs. A subset of biosensors operating in the radio frequency (RF) range of electromagnetic (EM) waves, called RF biosensors, offer appeal as a non-destructive, non-invasive form of sensing. A novel RF biosensor is proposed which detects changes in scattering parameter measurements of a microliter, aqueous material under test (MUT) held within a well adjacent to a microstrip transmission line. This sensing design measures scattering parameter data and changes in these measurements offer insight into the effects of RF wave exposure on dielectric materials within the well.

The following paper describes design considerations and the sensing technique of the proposed RF biosensor. Simulations were run in incremental steps to first, establish the simulation design of a 50-ohm microstrip transmission line using two software packages ADS and Ansys HFSS. Next, experimental measurements were collected by milling the RF biosensor, first using air and then distilled water as the MUT, and finally comparing to simulations to establish validity of the novel sensing device. Next, experimental S-parameter measurements were obtained and compared between the two test cases to determine if a difference could be detected. Both simulated and experimentally obtained measurements suggest the designed RF biosensor can detect changes in the MUT loaded inside its etched well and therefore can be used as a sensing device.
\end{abstract}

Keywords: RFBiosensor,Sensing Methods, Scattering Parameters

\section{INTRODUCTION}

Creating smaller, better, and faster diagnostic devices is a huge driving factor within the medical device industry. An area of current growth is designing radiofrequency (RF) biosensors for use in point of care testing (POCT) to diagnose and monitor patient health [1]. A strong benefit of these devices is that they have the potential to perform real-time analysis of the sample being tested. Also, RF waves are a non-ionizing form of electromagnetic energy, which means energy exposure is non-destructive and non- hazardous. Finally, RF biosensing can non-invasively perform diagnostic testing [2]. Even with these benefits, there is currently only one commercially available RF biosensor used for health care diagnostics.

Therefore, a need for a real-time, cost-efficient, and widely available RF biosensor for POCT exists [3-4]. The fundamental concepts behind the following design are as follows: If a threshold of EM energy is stored internally within the liquid, electric polarization can occur [5]. This phenomenon will align the charges internally within the liquid, which create changes in the dielectric property measurements of the material under test (MUT) [6-7]. Currently the industry standard for measuring dielectric property changes of aqueous MUT is equipment such as the Keysight High Temp Dielectric Probe. However, using this probe requires large volumes $(\sim \mathrm{mL})$ of MUT to perform testing. Getting this volume of aqueous material for samples that include proteins or DNA is not always feasible because of high cost or limited availability. 